\title{
SHORT COMMUNICATION \\ Papagaio-de-peito-roxo Amazona vinacea (Kuhl) (Aves: Psittacidae) no norte do Espírito Santo: redescoberta e conservação
}

\author{
Lucas A. Carrara; Luciene C. P. Faria; José R. Matos \& Paulo de Tarso Z. Antas
}

Fundação Pró-Natureza. SCLN 107, Bloco B, sala 201, 70743-520 Brasília, Distrito Federal, Brasil.

E-mail: lucascarrara@taskmail.com.br, lucienefaria@taskmail.com.br, matosroberto2004@yahoo.com.br; ptzantas@abordo.com.br

ABSTRACT. Vinaceous Amazon Amazona vinacea (Kuhl) (Aves: Psittacidae) in the northern region of Espirito Santo state, southeastern Brazil: rediscovery and conservation. Endemic of the Atlantic Forest, Amazona vinacea (Kuhl, 1820) is listed as endangered in Brazil and at the international level. Lack of recent reports north of Rio de Janeiro state, including the northern portion of Espírito Santo state (where it had been found probably till 1991) suggested the species had vanished from this part of the range. The present paper reports the rediscovery of $A$. vinacea in Alto Rio Novo region, northwestern Espírito Santo. Two flocks were seen in two different places, the largest one with 28 parrots was found in December 2005. The flocks of $A$. vinacea were eating seeds of Anadenanthera sp. (Fabaceae: Mimosoideae), a new resource for this parrot. Historical data list places up to $35 \mathrm{~km}$ from the two localities where the flocks were now found, stressing its regional importance for the Vinaceous Amazon. However, the continuous habitat degradation of the last forest remnants in the region and illegal capture of nestlings are considered the main constrain for this population's conservation. As recently as December 2002, the Pontões Capixabas National Park has been created. It is the first Conservation Unit in the mountains of northern Espírito Santo state. Luckily, it protects a historical place of the Vinaceous Amazon in the region and encompasses forested remnants suitable for the species. Its current limit is some $10 \mathrm{~km}$ apart from one of the places where the parrot was found in this study. Besides the conservation, data gathering on the Vinaceous Amazon populations in northwestern Espírito Santo state and the nearby region of Minas Gerais state are critical for the species future in the area. A management plan could be then established, aiming to conserve the current northernmost currently known population of this parrot.

KEY WORDS. Atlantic Forest; Conservation Units; food source.

RESUMO. Amazona vinacea (Kuhl, 1820) é uma espécie endêmica da Mata Atlântica e ameaçada de extinção no Brasil e no mundo. Não existem relatos recentes de sua presença na porção setentrional de sua distribuição ao norte do estado do Rio de Janeiro. O último é datado provavelmente de 1991, tendo sido considerado extinto ao norte do Espírito Santo. O presente trabalho noticia a redescoberta da espécie em Alto Rio Novo, noroeste do Espírito Santo, divisa com Minas Gerais. Foram registrados bandos em duas localidades em dezembro de 2005, sendo o maior deles composto por 28 indivíduos. Durante os registros foi observado $A$. vinacea se alimentando de Anadenanthera sp. (Fabaceae: Mimosoideae), uma nova fonte alimentar para a espécie. Os registros históricos mais recentes para a região citam localidades com distância inferior a $35 \mathrm{~km}$ dos atuais registros, reforçando a importância local. No entanto, a degradação ambiental e a captura ilegal representam obstáculos à conservação da espécie nesta região. Em dezembro de 2002 foi criado o Parque Nacional dos Pontões Capixabas, primeira Unidade de Conservação na porção serrana do norte do Espírito Santo. Uma das áreas históricas da espécie está incluída em seus limites e um dos locais onde foi agora redescoberto fica a cerca de $10 \mathrm{~km}$ de distância da borda do parque. Essa Unidade de Conservação é essencial à proteção de trechos nativos da Mata Atlântica e conseqüentemente do papagaio-depeito-roxo. Além de medidas conservacionistas, a aquisição de informações sobre as populações de $A$. vinacea no noroeste do Espírito Santo e leste de Minas Gerais torna-se indispensável para a proposição de medidas de manejo capazes de reverter o grave quadro atual, objetivando viabilizar a permanência de populações na região mais setentrional da distribuição conhecida atualmente para a espécie.

PALAVRAS-CHAVE. Fonte alimentar; Mata Atlântica; Unidades de Conservação.

O papagaio-de-peito-roxo Amazona vinacea (Kuhl, 1820) é uma espécie endêmica da Mata Atlântica, ocorrendo desde o estado da Bahia até o Rio Grande do Sul, além do leste do Paraguai e norte da Argentina (Sick 1997, Cockle et al. 2007). No entanto, sua ocorrência tornou-se fragmentada, limitando-se a esparsos e escassos fragmentos dentro da sua área de distribuição original

Revista Brasileira de Zoologia 25 (1): 154-158, March, 2008 
(Collar et al. 1992), justificando sua inclusão em praticamente todas as listas de espécies ameaçadas de extinção, independente da escala de abrangência. Amazona vinacea está sob risco de extinção no mundo (BirdLIFE InTERnATIONAL 2000), no Brasil (IBAMA 2003), em Minas Gerais (Machado et al. 1998), em São Paulo (São Paulo 1998), no Rio de Janeiro (Bergallo et al. 2000), no Espírito Santo (Espírito SANTO 2005) e no Rio Grande do Sul (MARQUEs et al. 2002). No estado do Paraná existem populações maiores, mesmo assim a espécie aparece na categoria de quase ameaçada (Мikich \& Bérnils 2004).

Considerada mais comum na região sul do Brasil, a ausência de registros recentes para os estados da Bahia (Cordeiro 2002) e Espírito Santo sugere uma forte retração de sua distribuição setentrional. Os últimos registros nesses estados para indivíduos em liberdade ocorreram em 1981 na Bahia (Collar et al. 1992) e, talvez, até 1990 no Espírito Santo (Fortaleza apud SiCK 1997). Apesar da proximidade das localidades capixabas, não existem registros históricos na região leste de Minas Gerais ao norte do rio Doce (Collar et al. 1992, Melo JR 1998).

No Espírito Santo, D. Fortaleza (apud Sick 1997) acompanhou o decréscimo acelerado da população do norte do estado a partir do final da década de 70, motivado pelo desmatamento das áreas nativas e retirada de filhotes. O extermínio da população foi previsto para 1983 e em 1990 não mais se encontrava A. vinacea na região (Fortaleza apud Sick 1997), tendo sido considerada extinta no norte do Espírito Santo (Collar et al. 1992).

O presente trabalho tem por objetivo noticiar a redescoberta de $A$. vinacea para o noroeste do estado do Espírito Santo, mais de 15 anos após os últimos relatos.

As informações aqui apresentadas foram obtidas ao longo do projeto de monitoramento da avifauna em propriedades privadas inscritas no Programa de Fomento Florestal desenvolvido pela empresa Aracruz Celulose S.A. na região serrana do estado do Espírito Santo. Iniciado em 2003, o monitoramento vem sendo realizado por meio de capturas, censos populacionais e inventário de espécies de aves, em duas etapas por ano, com duração em torno de 17 dias cada, abrangendo talhões de eucalipto e remanescentes de floresta nativa.

Em dezembro de 2005, registramos $A$. vinacea em duas localidades do município de Alto Rio Novo (500 a 750 metros de altitude), noroeste do estado. Um bando de $15 \mathrm{~A}$. vinacea foi avistado na manhã do dia oito na região sul do município, juntamente com algumas maitacas Pionus maximiliani (Kuhl, 1820). As duas espécies foram observadas se alimentando de sementes de angico Anadenanthera sp. (Fabaceae: Mimosoideae), árvore comum localmente, encontrada tanto em remanescentes como isolada em áreas transformadas em pastagens. A área de vegetação nativa na propriedade estudada estava restrita a um único fragmento de 4 ha, onde os papagaios permaneceram durante toda a manhã.

Dois dias depois, a espécie foi encontrada ao entardecer, por meio de registro visual e auditivo, aproximadamente a 5 $\mathrm{km}$ a noroeste da propriedade anterior, em um dos maiores fragmentos de floresta nativa da região (48 ha em diversos estádios sucessionais). Os registros se repetiram ao longo dos dois dias subseqüentes, sendo o maior bando formado por 28 indivíduos (contagem em vôo). A vocalização da espécie foi documentada através de gravador digital (Marantz, PMD650U e microfone Azden SGM-2X) e inserida no Arquivo Sonoro da Aracruz Celulose.

Assim como nas primeiras observações, os bandos de A.vinacea foram vistos utilizando os recursos da mesma espécie vegetal (Anadenanthera sp.). Análise detalhada das vagens e sementes revelou o consumo dos embriões e apenas parte dos cotilédones. As sementes consumidas estavam em processo final de amadurecimento.

Fontes alimentares de A.vinacea começaram a ser relatadas recentemente na porção sul de sua distribuição geográfica (COCKLE et al. 2007). Historicamente, associa-se a presença de $A$. vinacea à ocorrência do pinheiro-do-Paraná Araucaria angustifolia (CollaR et al. 1992), cuja semente é uma das fontes alimentares mais citadas. Porém, o limite setentrional de A. angustifolia é o sul dos estados de Minas Gerais e Espírito Santo (Lorenzi 1998), não alcançando o local do presente estudo. Além do pinheiro-doParaná, na porção meridional de sua ocorrência, A.vinacea utiliza inflorescências e sementes de outras coníferas (como Podocarpus lambertii e mesmo o exótico Pinus sp.), brotos, frutos e flores de várias espécies, incluindo da família Fabaceae (CockLE et al. 2007). Na região metropolitana de Curitiba há registros de alimentação de frutos de jerivá Syagrus romanzoffianum, araçá Psidium longipectiotalum, pitanga Eugenia uniflora, cerejeira Eugenia involucrata e flores de corticeira Erythrina falcata, papagaieira Laplacea fruticosa e bracatinga Mimosa scabrella (Аве 2000). Há também registros de alimentação de folhas novas de Eucalyptus sp. e Pinus sp., frutos de palmito Euterpe edulis, folhas e brotos de taquara Guadua sp., além de relatos históricos que mencionam ataques a pomares de laranja (Collar 1997). O atual registro trata-se, portanto, do primeiro uso comprovado desse gênero de leguminosa de ampla distribuição geográfica, podendo representar uma importante fonte alimentar para A. vinacea na região setentrional de sua distribuição durante pelo menos parte do ano.

Na província de Misiones, Argentina, esse papagaio mantém sua maior concentração populacional em região com ocupação humana semelhante a Alto Rio Novo, onde existem pequenas e médias propriedades rurais praticando agricultura e pecuária de subsistência. Por outro lado, no nordeste do Paraguai, onde a ocupação agropastoril intensa não deixa fragmentos florestais e nem mesmo árvores isoladas nas áreas derrubadas, a espécie está restrita aos remanescentes florestais mais extensos ainda existentes (COCKLE et al. 2007). Dentre os poucos monitoramentos conduzidos com a espécie no Brasil, foi acompanhado um grupo na região metropolitana de Curitiba utilizando uma faixa de Floresta Atlântica, remanescentes de Floresta com Araucária e também reflorestamentos com $P$. elliottii, sendo os últimos utilizados como dormitórios em determinada época do ano (AвE 2000). 

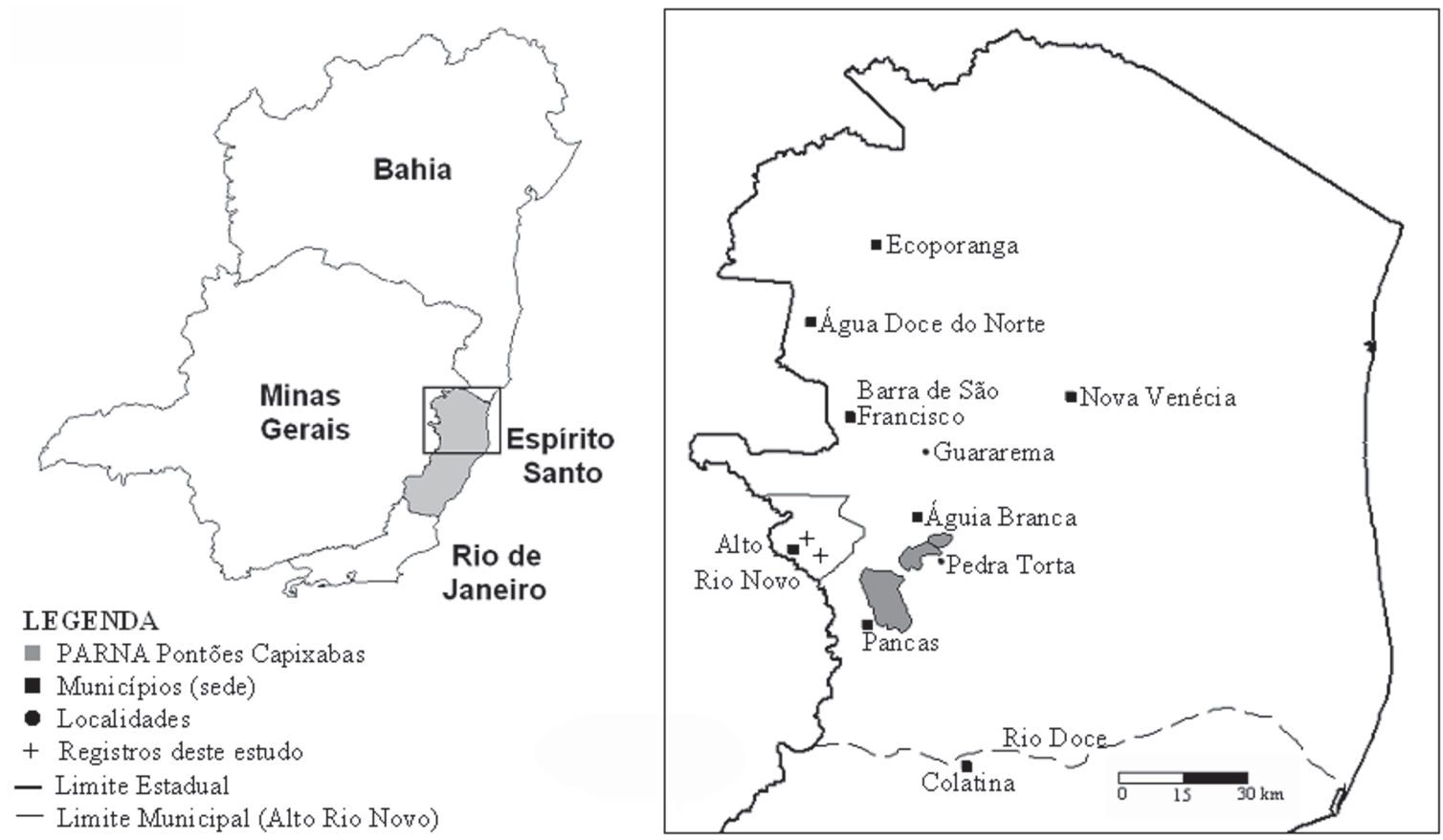

Figura 1. Localização dos registros de Amazona vinacea no Parque Nacional dos Pontões Capixabas e nos principais municípios do noroeste do Espírito Santo.

A concentração espacial e temporal dos registros de $A$. vinacea em Alto Rio Novo permite apenas especulações a respeito da existência de movimentos migratórios para espécie na região. Na literatura, as referências a movimentos dessa espécie são pouco precisas e contraditórias, indicando que ocasionalmente bandos grandes invadiam o Paraguai (Collar 1997). De acordo com Straube et al. (2004), A. vinacea realiza grandes deslocamentos sazonais no Paraná, podendo esse comportamento estar associado a variações anuais de produção de pinhão ou de outra fonte alimentar. Por outro lado, na região metropolitana de Curitiba, um bando de 500 indivíduos permaneceu no mesmo local ao longo de todo o ano (Aве 2000). No Rio Grande do Sul, há menção a um movimento de dispersão pós-reprodução, desaparecendo completamente em março e reaparecendo em abril para permanecer o resto do ano (Collar 1997). Entretanto, não há menção a tal movimento por BeLton (1994), o que torna a determinação de migrações ainda mais frágil. No extenso trabalho feito no Paraguai e Argentina de 1997 a 2006 não foram determinados movimentos migratórios nas duas populações estudadas (CocKLe et al. 2007). A ausência de informações para o leste de Minas Gerais também dificulta a situação, já que são registros exclusivamente de ocorrência. O registro mais próximo da área do Espírito Santo aqui relatada situa-se em Caratinga (Melo Jr 1998), ao sul do rio Doce e a $170 \mathrm{~km}$ a sudoeste da região de Alto Rio Novo. Sabe-se que o papagaio-charão Amazona pretrei (Temminck, 1830) realiza movimentos migratórios associados à oferta de pinhões de $A$. angustifolia no sul do país
(Martinez \& Prestes 2002). Estudos mais detalhados com as populações setentrionais de $A$. vinacea são necessários para determinar qualquer padrão de deslocamento estacional capaz de explicar a sazonalidade da observação de Alto Rio Novo.

Os registros históricos mais recentes disponíveis na literatura para o norte do estado citam Guararema e Pedra Torta (Collar et al. 1992), localidades situadas em um raio de até 35 $\mathrm{km}$ das propriedades citadas no presente relato. Em 1989 foram registrados em número bastante reduzido em Alto Rio Novo e Pancas (ForTaleza 1990). Registros anteriores relatam sua presença em Água Doce do Norte e Barra de São Francisco, municípios situados mais no norte do estado (Fig. 1).

A região noroeste do Espírito Santo representa, portanto, uma área de extrema importância para a conservação das populações mais setentrionais de $A$. vinacea conhecidas atualmente. No entanto, a região vem sofrendo há décadas com a destruição ambiental, principalmente motivada pela exploração madeireira, expansão agropecuária e, nos anos mais recentes, pela extração de granito nos remanescentes de Mata Atlântica.

Em dezembro de 2002 foi criado o Parque Nacional dos Pontões Capixabas, abrangendo uma área de 17.496 ha nos municípios de Pancas e Águia Branca (decreto sem número de 19 de dezembro de 2002). Seus limites ficam a apenas a $10 \mathrm{~km}$ de uma das localidades onde os bandos de $A$. vinacea foram avistados em 2005 (Fig. 1). A Pedra Torta, local histórico de ocorrência da espécie, está parcialmente englobada pela delimitação atual da unidade. 
A criação e a implementação desta Unidade de Conservação representam medidas essenciais à perpetuação de fragmentos nativos na porção serrana da região noroeste do estado, extremamente carente em áreas protegidas e conservadas. Não existe nenhuma outra Unidade de Conservação na região serrana do norte do estado, seja de proteção integral ou de uso sustentável (CONSERVATION INTERNATIONAL 2006), reforçando ainda mais a necessidade da efetivação do Parque Nacional dos Pontões Capixabas. A proteção de áreas de vegetação nativa representa uma medida básica para garantir a manutenção de populações viáveis de $A$. vinacea.

Além da proteção de remanescentes florestais, torna-se urgente o acompanhamento das populações de $A$. vinacea na região, tanto no noroeste do Espírito Santo quanto no leste de Minas Gerais. Apesar da ausência de registros nessa porção de Minas Gerais, sua possível ocorrência torna-se plausível devido à proximidade física da divisa com os locais onde os grupos foram observados e à capacidade de deslocamento da espécie. O acompanhamento detalhado da população redescoberta visa adquirir conhecimento da atual situação da espécie, mapear suas áreas de alimentação e reprodução, identificar fontes alimentares e sua fenologia, assim como determinar as principais limitações à sua manutenção e seu crescimento populacional. A aquisição destas informações será indispensável para se propor medidas de manejo que sejam capazes de reverter o grave quadro atual, sendo possível viabilizar a permanência de $A$. vinacea no estado do Espírito Santo e, por extensão, no leste de Minas Gerais.

\section{AGRADECIMENTOS}

Somos gratos à Aracruz Celulose S.A. e Fundação Pró-Natureza. Aos funcionários do programa de Fomento Florestal da Aracruz Celulose S.A., em especial Alex S. Lima e Jairo Dal'Col, também Vanir Tschaen, Ivan Nogueira, Wellinton Pinheiro e Brisa Moulin, responsáveis pelo apoio local. Agradecemos a Aroldo R. R. Junior e toda equipe do Centro de Pesquisas Tecnológicas (CPT) da empresa. Aos revisores anônimos pelas valiosas sugestões ao manuscrito. Aos proprietários das áreas de estudo pela permissão e interesse no desenvolvimento do projeto.

\section{LITERATURA CITADA}

AвE, L.M. 2000. Ecologia, distribuição e monitoramento de uma população de papagaio-de-peito-roxo (Amazona vinacea) na Região Metropolitana de Curitiba, Paraná, p. 226. In: F.C Straube; M.M. Argel-de-Oliveira \& J.F. CÂndido-Júnior (Eds). Ornitologia brasileira no Século XX. Curitiba, Editora Popular, 428p.

Belton, W. 1994. Aves do Rio Grande do Sul. Rio Grande do Sul, Editora UNISINOS, 584p.

Bergallo, H.G.; C.F.D. Rocha, M.A.S. Alves \& M.V. Sluys. 2000. A fauna ameaçada de extinção do estado do Rio de Janeiro. Rio de Janeiro, Editora UERJ, 166p.
Birdlife International. 2000. Threatened birds of the world. Barcelona, Lynx Edicions, Birdlife International, 852p.

Cockle, K.; G. Capuzzi; A. Bodrati; R. Clay; H. del Castillo; M. Velázquez; J.I. Areta; N. Fariña \& R. Fariña. 2007. Distribution, abundance, and conservation of Vinaceous Amazons (Amazona vinacea) in Argentina and Paraguay. Journal Field Ornithology 78 (1): 21-39.

Collar, N.J. 1997. Family Psittacidae (Parrots), p. 280-479. In: J. Del Hoyo; A. Elliot \& J. Sargatal (Eds). Handbook of the birds of the World. Barcelona, Lynx Edicions, 679p.

Collar, N.J.; L.P. Gonzaga; N. Krabbe; A. Madroño nieto; L.G. NARANJO; T.A. Parker III \& D. Wege.1992. Threatened birds of Americas: the ICBP/IUCN red data book. Cambridge, International Council for Bird Preservation, 1150p.

Conservation International. 2006. Unidades de conservação do Brasil. Disponível na World Wide Web em: http:// www.conservation.org.br [Acessado em 22/II/2006]

Cordeiro, P.H.C. 2002. A fragmentação da Mata Atlântica no sul da Bahia e suas implicações na conservação de psitacídeos, p. 215-227. In: M. Galetti \& M.A. Pizo (Eds). Ecologia e conservação de psitacídeos no Brasil. Belo Horizonte, Melopsittacus Publicações Científicas, 235p.

Espírito SANTO. 2005. Diário Oficial. Vitória, Imprensa Oficial do Estado do Espírito Santo, Poder Executivo [16/VI.2006]

Fortaleza, D.M.R. 1990. Observações dos psitacídeos do Espírito Santo e Sul da Bahia no período de acasalamento e reprodução. Atualidades Ornitológicas 35: 9.

Iвама. 2003. Lista das espécies de fauna brasileira ameaçada de extinção. Disponível na World Wide Web em: http:// www.ibama.gov.br/fauna/downloads/lista\%20spp.pdf [Acessado em 04/X/2005]

LoRENZI, H. 1998. Árvores brasileiras: manual de identificação e cultivo de plantas arbóreas nativas do Brasil. Nova Odessa, Editora Plantarum, vol. 1, 368p.

Machado, A.B.M.; G.A.B. Fonseca; R.B. Machado; L.M.S. Aguiar \& L.V. Lins. 1998. Livro vermelho das espécies ameaçadas de extinção da fauna de Minas Gerais. Belo Horizonte, Fundação Biodiversitas, 605p.

Marques, A.A.B.; C.S. Fontana; E. Vélez; G.A. Bencke; M. Schneider \& R.E. ReIs. 2002. Lista das espécies da fauna ameaçadas de extinção no Rio Grande do Sul. Porto Alegre, Publicações Avulsas Fundação Zoobotânica do Rio Grande do Sul, vol. 11, 52p.

Martinez, J. \& N.P. Prestes. 2002. Ecologia e conservação do papagaio-charão Amazona pretrei, p. 173-192. In: M. GALETTI \& M.A. Pizo (Eds). Ecologia e conservação de psitacídeos no Brasil. Belo Horizonte, Melopsittacus Publicações Científicas, 235p.

Melo JR, T. 1998. Amazona vinacea (Kuhl, 1820), p. 280-282. In: A.B.M Machado; G.A.B. Fonseca; R.B. Machado; L.M.S. Aguiar \& L.V. Lins (Eds). Livro vermelho das espécies ameaçadas de extinção da fauna de Minas Gerais. Belo Horizonte, Fundação Biodiversitas, 605p. 
Miкich, S.B. \& R.S. Bérnils. 2004. Livro vermelho da fauna ameaçada no estado do Paraná. Curitiba, Instituto Ambiental do Paraná, 763p.

São Paulo. 1998. Fauna ameaçada no estado de São Paulo. São Paulo, Secretaria do Meio Ambiente, Documentos Ambientais, Série Probio, 56p.

Received in 12.VI.2007; accepted in 04.III.2008.
SICK, H. 1997. Ornitologia brasileira. Rio de Janeiro, Editora Nova Fronteira, 862p.

Straube, F.C.; A. Urben-Filho \& D. Kajiwara. 2004. Aves, p. 145496. In: S.B. Мiкich \& R.S. Bérnils (Eds). Livro vermelho da fauna ameaçada no estado do Paraná. Curitiba, Instituto Ambiental do Paraná, 763p. 